\title{
The impact of warfarin on operative delay and 1-year mortality in elderly patients with hip fracture: a retrospective observational study
}

\author{
Gaetano Caruso ${ }^{1,2^{*}}$ (D), Mattia Andreotti ${ }^{3}$, Tedi Marko ${ }^{3}$, Francesco Tonon ${ }^{3}$, Nicola Corradi ${ }^{3}$, Damiano Rizzato ${ }^{3}$, \\ Alessandra Valentini ${ }^{4}$, Giorgia Valpiani ${ }^{5}$ and Leo Massari ${ }^{1,2}$
}

\begin{abstract}
Background: Guidelines underline the importance of early surgery in elderly patients with proximal femoral fractures. However, most of these patients present a high number of comorbidities, some of which require the use of warfarin. Waiting for INR decrease is a cause of surgical delay, and this influences negatively their outcome.

Methods: We retrospectively reviewed all patients with proximal femoral fracture admitted to our unit from March 2013 to March 2017 to determine whether warfarin therapy is associated with reduction of survival, delay of surgery, and increased blood loss. From 1706 patient, a total of 1292 fulfilled the eligibility criteria and were included. Data regarding general information (type of fracture according to AO/OTA classification), pharmacological history regarding anticoagulant therapy pre-admission, surgery (type of surgery and time to surgery), clinical findings (blood loss), and date of exitus were collected.

Results: We identified 157 patients with warfarin, 442 with antiplatelet agents (aspirin, clopidogrel, ticlopidin), and 693 in the control group. We observed a significant difference in the warfarin group regarding an increased ASA score, Charlson Comorbidity Index, and blood loss. Patients taking warfarin experience delay to the theater significantly more than the other groups. Patients in warfarin therapy have a $42 \%$ higher risk of death within 1 year from their surgery. Patients who underwent surgery after $48 \mathrm{~h}$ have 1.5 times higher risk of mortality with respect to the patients who underwent surgery within $48 \mathrm{~h}$.
\end{abstract}

Conclusion: Warfarin therapy at the time of proximal femoral fractures is associated with increased time to surgery, blood loss, and mortality.

Keywords: Proximal femoral fractures, Elderly patients, Warfarin, Surgical timing, Mortality

\section{Introduction}

Hip fractures are common orthopedic injury and are a major cause of morbidity and mortality in the elderly. One-year mortality after hip fracture ranges from 14 to $36 \%$ [1-3]. Delayed surgical treatment and pre-admission comorbidities are associated with an increased post-operative mortality [4, 5]. Current

\footnotetext{
* Correspondence: crsgtn@unife.it

${ }^{1}$ Department of Biomedical and Speciality Surgical Sciences University of Ferrara, Via Luigi Borsari 46, 44121 Ferrara, Italy

${ }^{2}$ Orthopaedic and Traumatology Unit, Sant'Anna University Hospital of Ferrara, Via Aldo Moro 8, 44124 Cona, Ferrara, Italy

Full list of author information is available at the end of the article
}

guidelines recommend surgery within $48 \mathrm{~h}$ [6]. However, the association between delayed surgery and mortality remains elusive because of strong confounding by comorbidity factors. Long-term anticoagulant treatment is frequently encountered in patients with proximal femoral fracture (PFF) and associated with surgical delay [7]. Despite this, there are no set national standards for management of drug-induced coagulopathy pre-operatively in the context of hip fractures.

Warfarin is the most widely prescribed anticoagulant and it has been estimated that $4-8 \%$ of these patients are in therapy with it in the UK [8]. Traditionally, 
warfarinised patients were considered high-risk patients due to increased bleeding and associated perioperative complications, and their management could cause surgical delays. The current clinical practice recommends to postpone surgery until a patient's international normalized ratio (INR) decreases below 1.5. The time taken to achieve a safe INR together with the presence of multiple comorbidities has the potential to delay surgical treatment [9]. One of the strategies to reduce the bleeding in these patients is to temporarily postpone the warfarin administration and then wait until the anticoagulant effect of the warfarin has subsided, the action of warfarin lies between 2 and 5 days [10].The use of vitamin $\mathrm{K}$ to reverse the effect of warfarin has been shown to be safe and effective in the context of PFF surgery even though there is no widely accepted consensus on the absolute indications, dosage, and route of vitamin $\mathrm{K}$ treatment in the context of hip fracture [11].

The role of the antiplatelet therapy (aspirin, clopidogrel, ticlopidin) in the management of elderly patients with hip fractures seems to be less relevant. A clinical study addressing the use of both aspirin and clopidogrel when surgery for hip fractures is not delayed concluded that there was no difference in bleeding complication, blood loss, or transfusion requirements, when compared with those not on antiplatelets [12].

More recently, the factor Xa inhibitors have been popularized (apixaban, dabigatran, and rivaroxaban) [13]. Common practice is to interrupt the factor Xa inhibitors for 24-48 h and undertake surgery thereafter [14]. Patients with PFF taking Xa inhibitors seem to have a longer delay in surgery compared to patients on warfarin or no anticoagulation [15].

The purpose of this study is to determine whether warfarin therapy at the time of admission for PFF is associated with reduction of survival, delay of surgery, and increased blood loss.

\section{Materials and methods}

This is a retrospective cross-sectional study on consecutive patients with PFF admitted between March 2013 and March 2017 at our Orthopedic and Traumatology Unit located in the northeast of Italy (catchment area which caters to roughly 350,000 inhabitants).

Patients older than 65-years undergoing surgery for PFF were identified retrospectively from a hospital discharge database. The fractures were classified according to the AO-Müller/Orthopaedic Trauma Association fracture and dislocation classification (AO/OTA) [16]. The inclusion criteria were to have isolated proximal femoral fracture 31-A1, 31-A2, 31-A3, 31-B1, 31-B2, and 31-B3 (according to AO/OTA classification) and to have been treated surgically with joint reconstruction (total or emi- hip arthroplasty) or fixation with intra- or extramedullary devices (proximal femoral plate or proximal femoral short and long nail) with a minimum follow-up of 12 months. Patients with pathological or atypical femoral fractures, or fractures treated with other fixation devices (fixation with screws); patients with factor Xa inhibitors therapy (apixaban, rivaroxaban, fondaparinux); and patients still alive that did not undergo a complete follow-up at 365 days post-operatively were excluded (Fig. 1).

Data regarding general information, pharmacological history, surgery, date of exitus, and clinical findings were collected. We calculated hours between admission and surgery for all patients using threshold of $48 \mathrm{~h}$ as suggested in literature [6]. The American Society of Anesthesiologists Physical Status Classification System score (ASA) and Charlson Comorbity Index (CCI) were extracted to evaluate patient comorbidities [17]. In order to estimate blood loss, we used the method of hemoglobin balance described by Foss and Kehlet. [18] We gathered preoperative and 3 days post-operative hemoglobin values and units of fresh packed red blood cell transfused, as this method expected. We registered mortality data at 30,180 , and 365 days for each group of patients.

In pharmacological data, we divided the whole sample in three groups based on the anticoagulant therapy that they took at the admission: warfarin, antiplatelet drugs (aspirin, clopidogrel, ticlopidine), and the control group contained patients that did not take any anticoagulant or antiplatelet agents. Patients on warfarin therapy underwent a discontinuation protocol as suggested by the British Society of Haemetalogy [19] that consists of using oral or intravenous vitamin $\mathrm{K}$ in order reduce the INR values to operate these patients within $48 \mathrm{~h}$. Patients were not treated with a standardized warfarin reversal protocol and received intravenous administration of a dose of $10 \mathrm{mg} / \mathrm{ml}$ of vitamin $\mathrm{K} 1$ (Konakion) maximum twice a day based on the INR values. INR values were checked at the admission of the patient and every $8 \mathrm{~h}$ until surgery. We also shared our groups in those whom underwent surgery within or after $48 \mathrm{~h}$, as well as by type of surgery to evaluate the potential association between warfarin treatment and time to surgery and surgical delay and mortality.

\section{Statistical analysis}

Continuous data were checked for normality using ShapiroWilk test. All continuous variables were expressed as means and standard deviation (SD) for normality or medians and interquartile ranges (IQR25-75) for nonnormally distributed continuous variables. Categorical data were expressed as total numbers and percentages. Statistical comparisons of continuous variables were performed using unpaired analysis of variance for normally distributed and nonparametric Kruskal-Wallis test for non-normally distributed variables, whereas 


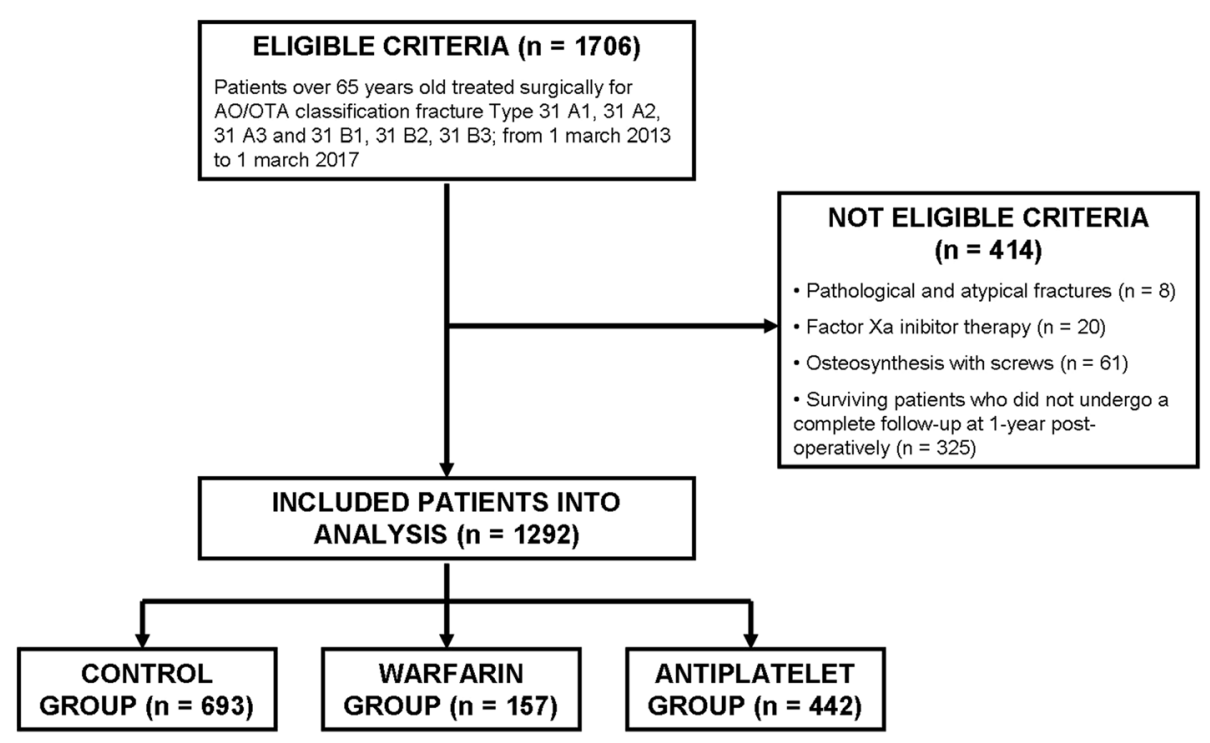

Fig. 1 Flowchart describing the inclusion and exclusion criteria of the study

categorical variables were assessed using Pearson's $\chi^{2}$ test. Survival curves estimated with Kaplan-Meier methods were compared via log-rank test among groups of anticoagulant therapy. In this case, mortality was assessed at 30 days, 180 days, and 365 days. Kaplan-Meier survival estimation model was performed for long-term survival up to 1-year follow-up comparing the impact of three different groups. Age, gender, age, anticoagulant therapy, ASA score, Charlson Comorbidity Index, AO/OTA Classification, types of surgical procedure, time to surgery, and blood loss were assessed as a prognostic factor for survival using Cox regression analysis [20, 21]. Additionally, a stepwise selection procedure based on likelihood-ratio test was performed. In backward elimination, we considered a removal probability equal to 0.10 . We analyze Schoenfeld residuals to test the assumption of proportional hazards [22]. For the time to event analysis, patients were censored at the time of death. Further, we calculated multivariate Cox regression analysis adjusted for potential confounders to assess which parameters had an independent association with mortality. Goodness of fit of the model is evaluated graphically by Cox-Snell residues. A two-sided $p$ value $<0.05$ was considered statistically significant. Statistical analysis was performed using STATA 12.1 (StataCorp, TX, USA).

\section{Results}

From 1706 patients admitted with hip fractures during the study period, a total of 1292 (76\%) patients fulfilled eligibility criteria and were included in the study, 414 (24\%) patients were excluded. We identified 157 (12\%) patients with warfarin, 442 (34\%) with antiplatelet agents, and 693
(54\%) in the control group. All demographics and clinical data of each group are summarized in Table 1. Comparing the data of the 3 groups (warfarin, antiplatelet, and control group), we noticed that the sample is composed by prevalently female patients (74\%) and there were no significant differences regarding age at the time of surgery. Half of the patients $(n=641)$ belong to the highest ASA risk classes (ASA 4/5). ASA score and Charlson Comorbidity Index were significantly higher in the warfarin group $(p<0.001)$. Regarding the time of surgery, $54 \%$ of the patients in the warfarin group underwent surgery after $48 \mathrm{~h}$, while the $63 \%$ of the patient in the antiplatelet group and the $69 \%$ of the patient in the control group underwent surgery within $48 \mathrm{~h}$, showing an opposite trend $(p<0.001)$. The median blood loss was significantly higher in the warfarin group $(p=0.0054)$. Mortality was higher in patients in warfarin group at 30 days $(p=0.0484)$, at 180 days $(p=0.0005)$, and at 1 year $(p<0.0001)$. No statistical difference was observed regarding type of fracture (according to AO/OTA classification) and type of surgery (joint reconstruction or fixation with intra- or extramedullary devices).

The Kaplan-Meier survival curve confirms that mortality of the patients in the warfarin group was significantly higher compared to the other groups (Table 1 and Fig. 2). In terms of the effect of the surgical delay on the survival rate, there are no significant differences in the total sample analyzed $(p=0.0654)$, in the control group $(p=0.1440)$, and in the warfarin one $(p=0.3034)$. Analyzing the Kaplan-Meier survival curves, there is a higher survival trend in patients who underwent surgery within $48 \mathrm{~h}$ (Fig. 3) but it is not statistically significant. 
Table 1 Demographic, clinical, and surgery-related characteristics of all patients

\begin{tabular}{|c|c|c|c|c|c|}
\hline & Warfarin & Antiplatelet & Control & Total & $p$ value \\
\hline Patient & $157(12 \%)$ & 442 (34\%) & $693(54 \%)$ & $1292(100 \%)$ & \\
\hline \multicolumn{6}{|l|}{ Gender } \\
\hline Male & $59(38 \%)$ & $115(26 \%)$ & 159 (23\%) & $333(26 \%)$ & \multirow[t]{2}{*}{0.001} \\
\hline Female & $98(62 \%)$ & 327 (74\%) & $534(77 \%)$ & 959 (74\%) & \\
\hline Age, mean $\pm s d$ & $84.76 \pm 6.08$ & $84.09 \pm 6.61$ & $83.69 \pm 8.15$ & $83.96 \pm 7.42$ & 0.3567 \\
\hline \multicolumn{6}{|l|}{ ASA score } \\
\hline $1 / 2$ & 0 & $13(3 \%)$ & $69(10 \%)$ & $82(7 \%)$ & \multirow[t]{3}{*}{$<0.001$} \\
\hline 3 & 34 (22\%) & 165 (38\%) & $350(51 \%)$ & $549(43 \%)$ & \\
\hline $4 / 5$ & $120(78 \%)$ & 256 (59\%) & 265 (39\%) & $641(50 \%)$ & \\
\hline $\mathrm{CCl}$, median (IQR, Q1 to Q3) & $6(5-7)$ & $6(4-7)$ & $5(4-6)$ & $5(4-7)$ & 0.0001 \\
\hline \multicolumn{6}{|l|}{ AO/OTA Classification } \\
\hline $\mathrm{A} 1$ & $42(27 \%)$ & 93 (21\%) & 139 (20\%) & $274(21 \%)$ & \multirow[t]{6}{*}{0.47} \\
\hline $\mathrm{A} 2$ & $34(22 \%)$ & $108(25 \%)$ & $164(24 \%)$ & $306(24 \%)$ & \\
\hline A3 & $7(4 \%)$ & $43(10 \%)$ & $65(10 \%)$ & $115(9 \%)$ & \\
\hline B1 & $20(13 \%)$ & $70(16 \%)$ & $112(16 \%)$ & $202(16 \%)$ & \\
\hline B2 & $43(28 \%)$ & $103(23 \%)$ & $168(24 \%)$ & $314(24 \%)$ & \\
\hline B3 & $10(6 \%)$ & $22(5 \%)$ & $43(6 \%)$ & $75(6 \%)$ & \\
\hline \multicolumn{6}{|l|}{ Types of surgical procedure } \\
\hline Reconstruction & $63(40 \%)$ & $178(40 \%)$ & $282(41 \%)$ & $523(40 \%)$ & \multirow[t]{2}{*}{0.986} \\
\hline Fixation & $94(60 \%)$ & $264(60 \%)$ & 411 (59\%) & 769 (60\%) & \\
\hline \multicolumn{6}{|l|}{ Time to surgery } \\
\hline$<48 \mathrm{~h}$ & $72(46 \%)$ & 277 (63\%) & 479 (69\%) & $828(64 \%)$ & \multirow[t]{2}{*}{$<0.001$} \\
\hline$>48 \mathrm{~h}$ & 85 (54\%) & $165(37 \%)$ & $214(31 \%)$ & $464(36 \%)$ & \\
\hline \multicolumn{6}{|l|}{ Blood loss (ml) } \\
\hline Median (IQR, Q1 to Q3) & 964.04 (446.95-1652.62) & 904.105 (454.4-1486.93) & 864.3 (394.59-1349.29) & 879.02 (419.33-1446.43) & 0.0054 \\
\hline $0-490 \mathrm{ml}$ & $40(26 \%)$ & 119 (27\%) & $241(35 \%)$ & 400 (31\%) & \multirow[t]{4}{*}{0.033} \\
\hline $491-980 \mathrm{ml}$ & $40(26 \%)$ & 139 (31\%) & 197 (28\%) & $376(29 \%)$ & \\
\hline $981-1470 \mathrm{ml}$ & 30 (19\%) & 69 (16\%) & $96(14 \%)$ & $195(15 \%)$ & \\
\hline$>1471 \mathrm{ml}$ & $45(29 \%)$ & $115(26 \%)$ & 157 (23\%) & $317(25 \%)$ & \\
\hline \multicolumn{6}{|l|}{ Mortality } \\
\hline 30 days & $13(8 \%)$ & $14(3 \%)$ & $28(4 \%)$ & $55(4 \%)$ & $0.0484^{*}$ \\
\hline 180 days & $42(27 \%)$ & $69(16 \%)$ & $100(14 \%)$ & $211(16 \%)$ & $0.0005^{*}$ \\
\hline 1 year & 61 (39\%) & $109(25 \%)$ & $140(20 \%)$ & $310(24 \%)$ & $<0.0001^{*}$ \\
\hline
\end{tabular}

Note: *the $p$ values related to mortality at 30 days, 180 days, and 1 year were calculated with the log-rank test

The same consideration can be made for the control group (Fig. 4) and for the warfarin group (Fig. 5).

Cox multivariate regression analysis showed an increased risk of mortality in patients on warfarin therapy (Table 2), in particular, patients in warfarin therapy have a $42 \%$ higher risk of death within 1 year from surgery compared to a patient of the Control group (HRadj =1.42; CI 95\% 1.03-1.95). The patients of the antiplatelet group did not show any significant risk (HRadj $=0.95$; CI 95\% 0.73-1.23). The patients over 95 years who underwent surgery for a proximal femoral fracture have approximately 3 times higher risk of dying within 1 year compared to patients younger than 75 (HRadj $=2.96$; CI 95\% 1.65-5.31). As expected, the comorbidities number negatively influenced the survival of these patients. The risk of mortality increases of $24 \%$ for every single value of the CCI (HRadj $=1.24$; CI 95\% $1.17-1.32)$. Regarding time of surgery, patients who underwent surgery after $48 \mathrm{~h}$ have 1.5 times higher risk of mortality with respect to patients who 


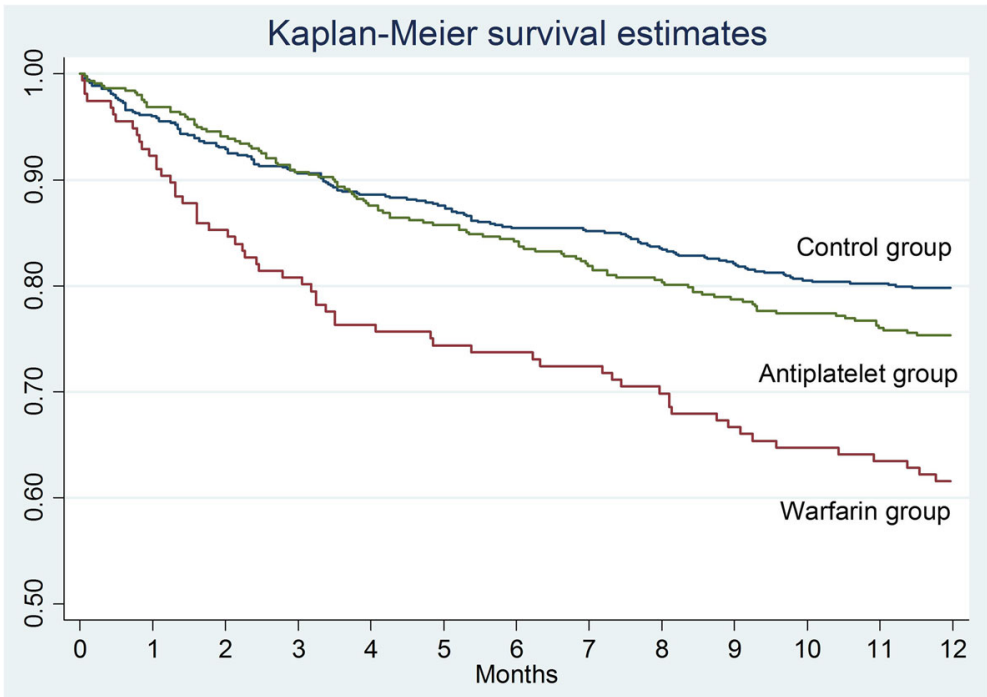

Fig. 2 The survival curves show the effect of warfarin treatment status on survival

underwent surgery within $48 \mathrm{~h}$, with equal other values (HRadj = 1.53; CI 95\% 1.04-2.25).

Patients who have a blood loss greater than $1471 \mathrm{ml}$ have a risk of death that is almost twice as high as those who have a blood loss lower than $491 \mathrm{ml}(\operatorname{HRadj}=1.81$; CI 95\% 1.30-2.53).

\section{Discussion}

Nowadays, most recent guidelines underline the importance of early surgery in elderly patients with PFF. However, most of these patients present a high number of comorbidities, some of which require the use of warfarin. These comorbidities and waiting time to achieve a safe
INR could cause a surgical delay. We found that warfarinised patients showed higher surgical delay and decreased survival.

Current guidelines suggest to operate these patients when INR values are $<1.5$ [13]. Different protocols have been proposed to reach these INR values as soon as possible by using Vitamin $\mathrm{K}$ agonists and derivatives, and it has demonstrated that they are safe procedures [23]. Mattison et al. in a recent case-control study analyzed 198 patients with extracapsular PFF and reported that using Vit.K and/or four-factor prothrombin complex concentrate to reach INR values $\leq 1.5$ in patients on warfarin therapy is safe [24]. Despite this, Bhatia et al.

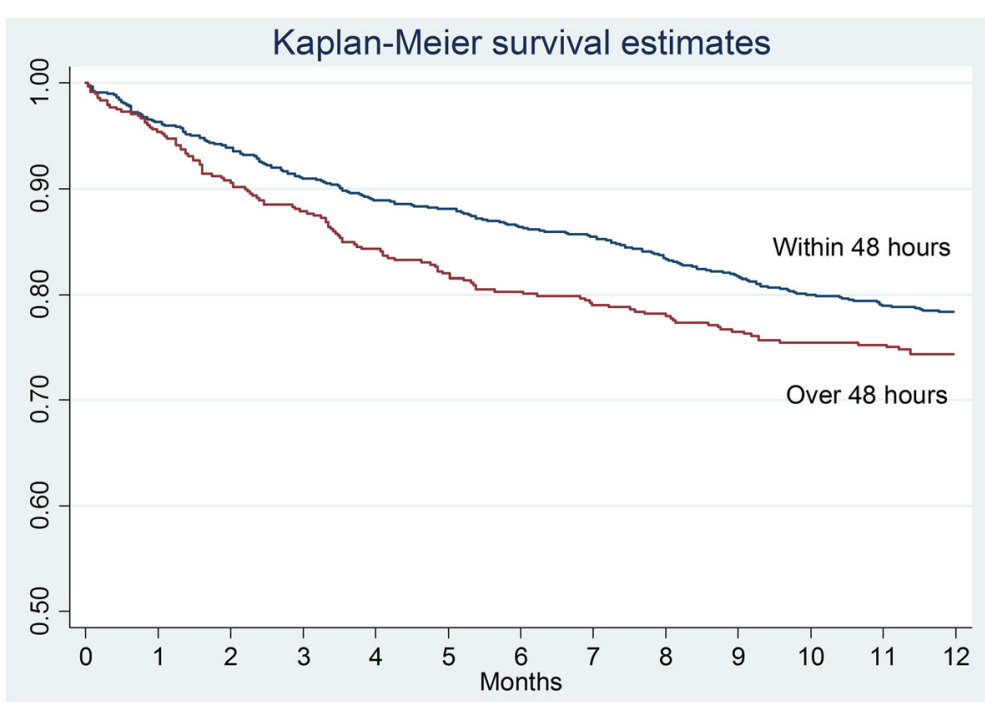

Fig. 3 The survival curves show the effect of surgical delay on survival 


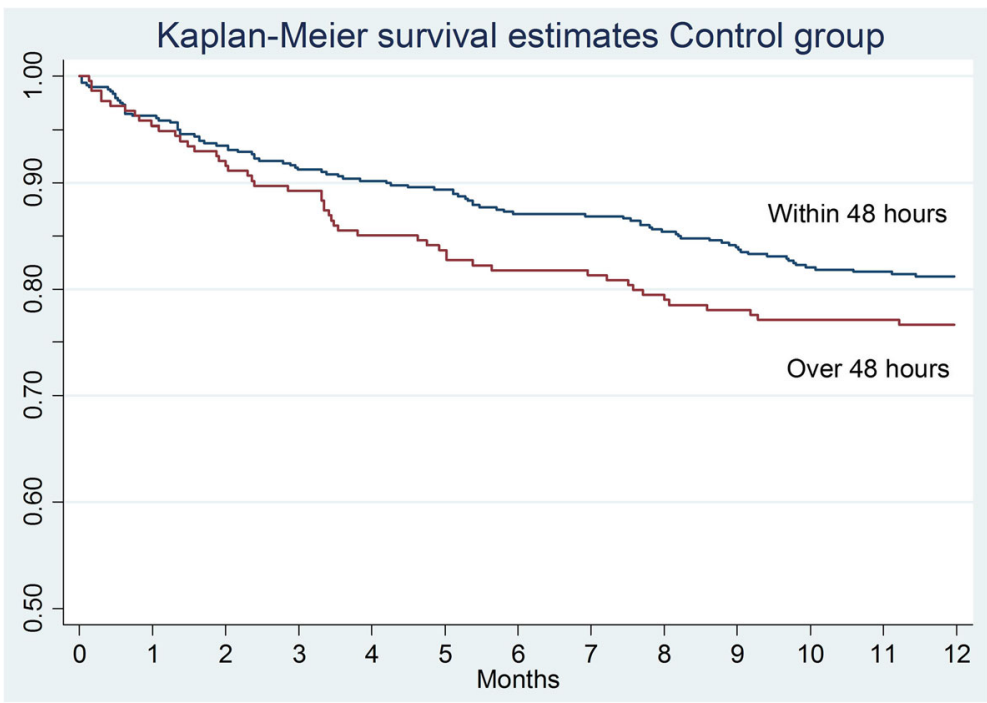

Fig. 4 The survival curves show the effect of surgical delay on survival in the control group

reported a time to surgery of at least 2 days (mean 38 hrs) in the warfarin group [25]. A study by Ryan et al. showed that surgical delay of more than $48 \mathrm{~h}$ resulted in higher mortality in patients with PFF, with an OR of 1.13 for the delayed group after controlling for multiple prognostic factors including comorbidity [26].

Even in our study, warfarinised patients showed higher mortality compared to patients that do not assume any anticoagulant drugs or antiplatelet therapy. In an observational study by Dettoni et al. published in 2011 including 875 patients with PFF, they noticed that warfarin therapy and delay in time to surgery were associated with a significantly higher risk for complications and mortality compared to all other patients [27]. Lawrence et al. showed an association between warfarin therapy with prolonged time to surgery and an increased 1-year mortality rate in hip fracture patients and they suggested to consider warfarin therapy a "Red flag" in patients with PFF [28]. Rosso et al. found out a 365 -day mortality of $15.4 \%$ in patients who underwent surgery within $48 \mathrm{~h}, 18.1 \%$ between 48 and 72 $\mathrm{h}$, and $21 \%$ over $72 \mathrm{~h}$ [29]. Recently, Moores et al. [30], in a retrospective analysis of prospectively collected matched data, evaluate post-operative mortality and lengh of stay in hospital for warfarinised hip fracture patients and compare them with a matched nonwarfarinised group, before and after the introduction of a standardized warfarin reversal protocol. They observed that the time of the somministration of the first dose of vitamin $\mathrm{K}$ is crucial for the

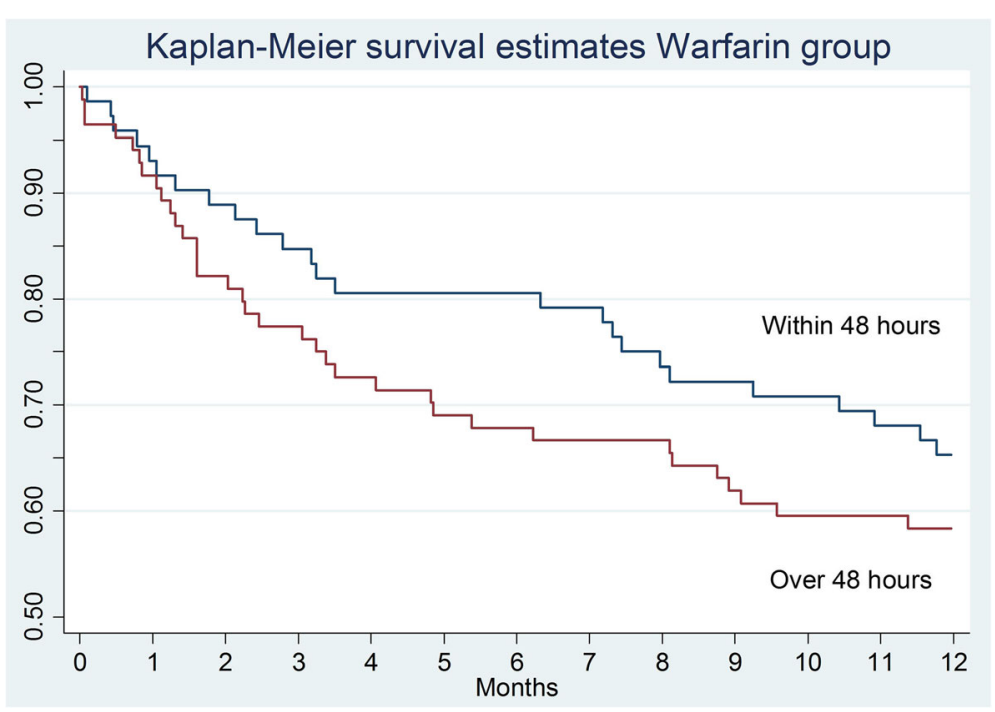

Fig. 5 The survival curves show the effect of surgical delay on survival in the warfarin group 
Table 2 Association between baseline patient characteristics and mortality according to Cox regression analysis, unadjusted and adjusted for potential confounders

\begin{tabular}{|c|c|c|c|c|c|c|}
\hline & \multicolumn{3}{|c|}{ Unadjusted Cox regression model } & \multicolumn{3}{|c|}{ Adjusted Cox regression model } \\
\hline & Crude HR & $\mathrm{Cl} 95 \%$ & $p$ value & Adjusted HR & $\mathrm{Cl} 95 \%$ & $p$ value \\
\hline \multicolumn{7}{|l|}{ Age (ref: 65-74yrs) } \\
\hline $75-84$ yrs & 1.13 & $0.69-1.84$ & 0.633 & 0.88 & $0.53-1.45$ & 0.621 \\
\hline $85-94$ yrs & 2.27 & $1.43-3.61$ & 0.001 & 1.48 & $0.92-2.39$ & 0.107 \\
\hline$\geq 95 \mathrm{yrs}$ & 4.33 & $2.47-7.57$ & $<0.001$ & 2.96 & $1.65-5.31$ & $<0.001$ \\
\hline \multicolumn{7}{|l|}{ Gender (ref: male) } \\
\hline Female & 0.63 & $0.50-0.80$ & $<0.001$ & 0.71 & $0.55-0.90$ & 0.005 \\
\hline \multicolumn{7}{|c|}{ Therapy (ref: control group) } \\
\hline Warfarin & 2.15 & $1.59-2.92$ & $<0.001$ & 1.42 & $1.03-1.95$ & 0.034 \\
\hline Antiplatelet & 1.23 & $0.96-1.58$ & 0.10 & 0.95 & $0.73-1.23$ & 0.687 \\
\hline \multicolumn{7}{|l|}{ ASA score (ref: 1/2) } \\
\hline 3 & 3.27 & $1.20-8.91$ & 0.021 & 1.72 & $0.62-4.79$ & 0.300 \\
\hline $4 / 5$ & 7.79 & 2.90-20.96 & $<0.001$ & 2.46 & $0.87-6.92$ & 0.089 \\
\hline $\mathrm{CCl}$ & 1.32 & $1.26-1.39$ & $<0.001$ & 1.24 & $1.17-1.32$ & $<0.001$ \\
\hline \multicolumn{7}{|c|}{ Time to surgery (ref: $<48 \mathrm{~h}$ ) } \\
\hline$>48 \mathrm{~h}$ & 1.24 & $0.99-1.56$ & 0.066 & 1.53 & $1.04-2.25$ & 0.032 \\
\hline \multicolumn{7}{|c|}{ Blood loss (ref: 0-490 ml) } \\
\hline $491-980 \mathrm{ml}$ & 1.92 & $1.38-2.66$ & $<0.001$ & 1.60 & $1.14-2.23$ & 0.006 \\
\hline $981-1470 \mathrm{ml}$ & 1.97 & $1.36-2.88$ & $<0.001$ & 1.38 & $0.94-2.04$ & 0.097 \\
\hline$>147 \mathrm{ml}$ & 2.60 & $1.88-3.59$ & $<0.001$ & 1.81 & $1.30-2.53$ & $<0.001$ \\
\hline \multicolumn{7}{|c|}{ AO/OTA Classification (ref: lateral fracture-A) } \\
\hline Medial fracture-B & 0.89 & $0.71-1.12$ & 0.332 & & & \\
\hline \multicolumn{7}{|c|}{ Types of surgical Procedure (ref: fixation) } \\
\hline Reconstruction & 0.93 & $0.74-1.17$ & 0.515 & & & \\
\hline
\end{tabular}

delay in warfarin reversal. They notice that somministration of the first dose decreases from 13 to $3 \mathrm{~h}(p<0.001)$ after protocol introduction, with all patients' INR being in a safe operative range within $24 \mathrm{~h}$ of admission.

Furthermore, in our study, we observed that the warfarin patient group had a greater blood loss than the other groups. This result differs from what is reported in Literature [24, 25, 31, 32].

Mattison et al. reported that there were no significant differences in terms of blood loss $(p=0.5)$; meanwhile, the number of units of red blood cell transfused was higher in the non-warfarin group ( $p=0.03)$ [24]. Cohn et al. in their study showed that $58.1 \%$ of the patients in warfarin underwent transfusion after surgery comparing to a $56.6 \%$ in the control group $(p=0.86)$. Also, the blood loss was similar in the two groups $(p=0.95)$ [33]. Even Lott et al. in their study of 479 patients with PFF, in which 112 were in therapy with warfarin, did not notice statistical differences in the two groups in terms of blood loss $(p=0.35)$ and post-operative transfusions $(p=0.535)$ [34].

For these reasons, the PFF NICE guidelines recommend to perform surgery "...on the day of, or the day after admission..." [35]. It is strongly indicated to identify and treat correctable comorbidities that may delay surgery, including anticoagulation therapy. Proactive anticoagulant management and expedient surgery reduce morbidity and mortality when managing this surgically challenging subset of hip fracture patients.

The main limitation of this study is the retrospective design. A randomized control trial would be more suitable to investigate this topic. However, the current knowledge and the guidelines would hardly ethically allow a study that compares early and late surgery in these patients. For this reason, we cannot be certain that awaiting INR was the only factor leading to delay of surgery and we were also unable to obtain specific data on post-operative complications including wound issues, thrombotic events, or pressure sores and causes of death. Additionally, we know the ASA grade, which reflects the comorbidities and the probability of medical complications, but we do not know precisely what these comorbidities are. Therefore, both the antagonization of the warfarin and the investigation and stabilization of these complications could have caused the surgical delay. Furthermore, due to the lack of a 
standardized institutional protocol, the decision to use warfarin inversion agents at variable times or to wait for INR normalization has the potential to bias. Despite these limitations, our results are consistent with previously published literature.

\section{Conclusions}

Our study confirmed the association between warfarin therapy at the time of admission for hip fracture, time to surgery, and survival rate. Despite the use of vitamin K and four-factor prothrombin complex for a fast reversal of the warfarin effect, waiting for INR values $\leq 1.5$ leads to a surgical delay. Surgical delay and comorbidities are the main reasons of the highest mortality of these fragile patients, the only possibility to increase survival rate of these patients is to identify and treat correctable comorbidities and to normalize INR value as soon as possible. For this reason, it is important to implement protocols that are finalized to decrease INR values for a faster surgery, starting from the admission of the patients with PFF on warfarin therapy in the Emergency Department.

\section{Abbreviations \\ AO/OTA: AO-Müller/Orthopaedic Trauma Association fracture and dislocation classification; ASA: Society of Anesthesiologists Physical Status Classification System score; CCl: Charlson Comorbity Index; Cl: Confidence interval; HR: Hazard ratio; INR: International normalized ratio; PFF: Proximal femoral fracture; SD: Standard deviation}

\section{Acknowledgement}

Monica Fedrizzi (acquisition of data) and Elisa Maietti (statistical processing of data).

\section{Funding}

None of the authors have received or will receive benefits for personal or professional use from a commercial party related directly or indirectly to the subject of this article.

\section{Availability of data and materials}

The datasets used and/or analyzed during the current study are available from the corresponding author on reasonable request.

\section{Authors' contributions}

GC reviewed the literature, conception and design, analysis and interpretation of data, and drafted the manuscript. MA, TM, FT, NC, and DR reviewed the literature and contributed to the conception and design, acquisition of data, and manuscript proofing. AV and GV contributed to the analysis and interpretation of the data. LM critical revised of the manuscript. All authors read and approved the final manuscript.

\section{Ethics approval and consent to participate}

The study was approved by the local Ethics Committee (Comitato Etico di Area Vasta Emilia Centro Italia) on April 18, 2018 (CE-AVEC 201/2018/Oss/AOUFe). Written informed consent was obtained from patients, and data collection and analysis was performed in compliance with the Declaration of Helsinki.

\section{Consent for publication}

Not applicable.

\section{Competing interests}

The authors declare that they have no competing interests.

\section{Publisher's Note}

Springer Nature remains neutral with regard to jurisdictional claims in published maps and institutional affiliations.

\section{Author details}

'Department of Biomedical and Speciality Surgical Sciences University of Ferrara, Via Luigi Borsari 46, 44121 Ferrara, Italy. ${ }^{2}$ Orthopaedic and Traumatology Unit, Sant'Anna University Hospital of Ferrara, Via Aldo Moro 8, 44124 Cona, Ferrara, Italy. ${ }^{3}$ Department of Morphology, Surgery and Experimental Medicine University of Ferrara, Via Luigi Borsari 46, 44121 Ferrara, Italy. ${ }^{4}$ Student in Statistical Sciences at University of Bologna, Via Zamboni 33, 40126 Bologna, Italy. ${ }^{5}$ Research and Innovation Office, Sant'Anna University Hospital of Ferrara, Via Aldo Moro 8, 44124 Cona, Ferrara, Italy.

Received: 16 October 2018 Accepted: 15 May 2019

Published online: 04 June 2019

\section{References}

1. Hu F, Jiang C, Shen J, Tang P, Wang Y. Preoperative predictors for mortality following hip fracture surgery: a systematic review and meta-analysis. Injury. 2012;43:676-85.

2. Smith T, Pelpola K, Ball M, Ong A, Myint PK. Pre-operative indicators for mortality following hip fracture surgery: a systematic review and metaanalysis. Age Ageing. 2014;43:464-71.

3. Schnell S, Friedman SM, Mendelson DA, Bingham KW, Kates SL. The 1-Year mortality of patients treated in a hip fracture program for elders. Geriatr Orthop Surg Rehabil. 2010;1:6-14

4. Shiga T, Wajima Z, Ohe Y. Is operative delay associated with increased mortality of hip fracture patients? Systematic review, meta-analysis, and meta-regression. Can J Anaesth. 2008;55(3):146-54.

5. Stoddart J, Horne G, Devane P. Influence of preoperative medical status and delay to surgery on death following a hip fracture. ANZ I Surg. 2002;72(6):405-7.

6. British Orthopaedic Association, British Geriatrics Society. The blue book: the care of patients with fragility fracture. 2007.

7. Yassa R, Khalfaoui MY, Hujazi I, Sevenoaks H, Dunkow P. Management of anticoagulation in hip fractures: a pragmatic approach. EFORT Open Rev. 2017;2(9):394-402.

8. Al-Rashid M, Parker MJ. Anticoagulation management in hip fracture patients on warfarin. Injury. 2005:36(11):1311-5.

9. Gleason $L$, Mendelson DA, Kates SL, Friedman SM. Anticoagulation management in individuals with hip fracture. J Am Geriatr Soc. 2014; 62(1):159-64

10. White RH, McKittrick T, Hutchinson R, Twitchell J. Temporary discontinuation of warfarin therapy: changes in the international normalized ratio. Ann Intern Med. 1995;122(1):40-2.

11. Buecking B, Eschbach D, Bliemel C, Oberkircher L, Struewer J, Ruchholtz S, Sachs UJ. Effectiveness of vitamin $\mathrm{K}$ in anticoagulation reversal for hip fracture surgery--a prospective observational study. Thromb Res. 2014; 133(1):42-7.

12. Collinge CA, Kelly KC, Little B, Weaver T, Schuster RD. The effects of clopidogrel (Plavix) and other oral anticoagulants on early hip fracture surgery. J Orthop Trauma. 2012;26(10):568-73.

13. Kailas SD, Thambuluru SR. Efficacy and safety of direct oral anticoagulants compared to warfarin in prevention of thromboembolic events among elderly patients with atrial fibrillation. Cureus. 2016;8(10):e836.

14. Heidbuchel H, Verhamme P, Alings M, Antz M, Diener HC, Hacke W, Oldgren J, Sinnaeve P, Camm AJ, Kirchhof P, ESC Scientific Document Group. Updated European Heart Rhythm Association practical guide on the use of non-vitamin-K antagonist anticoagulants in patients with non-valvular atrial fibrillation: executive summary. Eur Heart J. 2017; 38(27):2137-49

15. Tran T, Delluc A, de Wit C, Petrcich W, Le Gal G, Carrier M. The impact of oral anticoagulation on time to surgery in patients hospitalized with hip fracture. Thromb Res. 2015;136(5):962-5.

16. Meinberg EG, Agel J, Roberts CS, Karam MD, Kellam JF. Fracture and dislocation classification compendium-2018. J Orthop Trauma. 2018; 32(Suppl 1):S1-S170.

17. Charlson ME, Pompei P, Ales KL, MacKenzie CR. A new method of classifying prognostic comorbidity in longitudinal studies: development and validation. J Chronic Dis. 1987:40(5):373-83.

18. Foss NB, Kehlet $\mathrm{H}$. Hidden blood loss after surgery for hip fracture. J Bone Joint Surg Br. 2006;88:1053-9. 
19. Keeling D, Baglin T, Tait C, Watson H, Perry D, Baglin C, Kitchen S, Makris M, British Committee for Standards in Haematology. Guidelines on oralanticoagulation with warfarin - fourth edition. $\mathrm{Br} J$ Haematol. 2011; 154(3):311-24.

20. Harrell FE. Regression modelling strategies. Second ed: Springer; 2015. p. 399-533.

21. Hosmer DW, Lemeshow S. Applied survival analysis: regression modeling of time-to-event data: Wiley; 2008.

22. Schoenfeld D. Partial residuals for the proportional hazards regression model. Biometrika. 1982;69:239-41.

23. Ahmed I, Khan MA, Nayak V, Mohsen A. An evidence-based warfarin management protocol reduces surgical delay in hip fracture patients. J Orthop Traumatol. 2014;15(1):21-7.

24. Mattisson $L$, Lapidus $L$, Enocson $A$. Is fast reversal and early surgery (within $24 \mathrm{~h}$ ) in patients on warfarin medication with trochanteric hip fractures safe? A case-control study. BMC Musculoskelet Disord. 2018;19(1):203.

25. Bhatia M, Talawadekar G, Parihar S, Smith A. An audit of the role of vitamin $K$ in the reversal of international normalised ratio (INR) in patients undergoing surgery for hip fracture. Ann R Coll Surg Engl. 2010;92(6):473-6.

26. Ryan DJ, Yoshihara H, Yoneoka D, Egol KA, Zuckerman JD. Delay in hip fracture surgery: an analysis of patient-specific and hospital-specific risk factors. J Orthop Trauma. 2015:29(8):343-8.

27. Dettoni F, Castoldi F, Giai Via A, Parisi S, Bonasia DE, Rossi R. Influence of timing and oral anticoagulant/antiplatelet therapy on outcomes of patients affected by hip fractures. Eur J Trauma Emerg Surg. 2011;37:511-8.

28. Lawrence JE, Fountain DM, Cundall-Curry DJ, Carrothers AD. Do patients taking warfarin experience delays to theatre, longher hospital stay, and poorer survival after hip fracture? Clin Orthop Relat Res. 2017:475(1):273-9.

29. Rosso F, Dettoni F, Bonasia DE, Olivero F, Mattei L, Bruzzone M, Marmotti A, Rossi R. Prognostic factors for mortality after hip fracture: operation within 48 hours is mandatory. Injury. 2016;47 Suppl 4:S91-7.

30. Moores TS, Chatterton BD, Walker MJ, Roberts PJ. Standardised warfarin reversal expedites time to theatre for fractured neck of femur surgery and improves mortality rates: a matched cohort study. Advances in Orthopedics. 2018:2018:4791214.

31. Kumar D, Mbako AN, Riddick A, Patil S, Williams P. On admission haemoglobin in patients with hip fracture. Injury. 2011:42(2):167-70.

32. Smith GH, Tsang J, Molyneux SG, White TO. The hidden blood loss after hip fracture. Injury. 2011;42(2):133-5.

33. Cohn MR, Levack AE, Trivedi NN, Villa JC, Wellman DS, Lyden JP, Lorich DG, Lane JM. The hip fracture patient on warfarin: evaluating blood loss and time to surgery. J Orthop Trauma. 2017;31(8):407-13.

34. Lott A, Haglin J, Belayneh R, Konda SR, Leucht P, Egol KA. Does use of oral anticoagulants at the time of admission affect outcomes following hip fracture. Geriatr Orthop Surg Rehabil. 2018;9:2151459318764151.

35. Chesser TJ, Handley R, Swift C. New NICE guideline to improve outcomes for hip fracture patients. Injury. 2011;42(8):727-9.

Ready to submit your research? Choose BMC and benefit from:

- fast, convenient online submission

- thorough peer review by experienced researchers in your field

- rapid publication on acceptance

- support for research data, including large and complex data types

- gold Open Access which fosters wider collaboration and increased citations

- maximum visibility for your research: over $100 \mathrm{M}$ website views per year

At BMC, research is always in progress.

Learn more biomedcentral.com/submissions 